\title{
Bilateral Frontoparietal Polymicrogyria
}

National Cancer Institute

\section{Source}

National Cancer Institute. Bilateral Frontoparietal Polymicrogyria. NCI Thesaurus. Code C148367.

An autosomal recessive condition caused by mutation(s) in the ADGRG1 gene, encoding adhesion G-protein coupled receptor G1. It is characterized by motor and cog nitive developmental delay, pyramidal signs, and seizures. 